\title{
COULOMB INTERACTIONS IN THE EQUATION OF STATE
}

\author{
W. STOLZMANN AND T. BLÖCKER \\ Institut für Theoretische Physik und Sternwarte der Universität Kiel, \\ Olshausenstr. 40, W-2300 Kiel, Germany
}

\section{INTRODUCTION}

Various models are used in order to develop an accurate equation of state (EOS) for stellar matter (see e.g. Saumon and Chabrier 1991). The complexity of physical effects, for instance degeneracy, relativity, Coulomb- and non-Coulombic interactions requires detailed knowledge from the many-particle physics.

For many applications it is desirable to have theories for these effects over a wide range of densities and temperatures. One of the main sources of uncertainties in the EOS models is the exchange-correlation contribution at any degeneracy and relativity. Previous investigations determine thermodynamic quantities of a partially (e.g. Graboske et al. 1969, Kraeft et al. 1986, Däppen et al. 1988) or fully ionized (e.g. Hubbard and DeWitt 1985, Ichimaru et al. 1987) plasma, resp. Interpolation procedures (Magni and Mazzitelli 1979, Fontaine et al. 1977) combine both cases in the EOS but show non-continuous behaviour.

We present an EOS for fully ionized stellar matter which includes nonideal effects due to exchange-correlation interactions of charged particles at any degeneracy and which is applicable to arbitrary mixtures.

\section{EQUATION OF STATE}

For the ion-ion and the ion-electron terms we adopt the Padè formulae of Ebeling (1990). The Padè approximations consider the effect of weak (hydrogen-like) and strong (heavy ions) charge asymmetry. The electron-electron interaction is given by Ebeling et al. (1981) which we have modified at high densities.

Concerning the high density region in which relativistic effects are dominant the standard EOS of Salpeter (1961) is an excellent description at $T=0 \mathrm{~K}$. Finite temperature corrections of the high density EOS are given approximately for the exchange pressure by Shaviv and Kovetz (1972). We calculate the electronic exchange pressure in a consistent manner, valid for any degeneracy and relativity. The consideration of these relativistic corrections for the Coulombic part of the EOS closes the gap between the low-to-moderate density (non-relativistic) and the high density (relativistic) region for any temperatures.

\section{$\underline{\text { RESULTS }}$}

The figures show the relative pressure (ratio of total pressure to ideal pressure $P^{\text {id) }}$ vs. density along representative isotherms. $P^{\text {coul }}$ is the Coulomb contribu- 


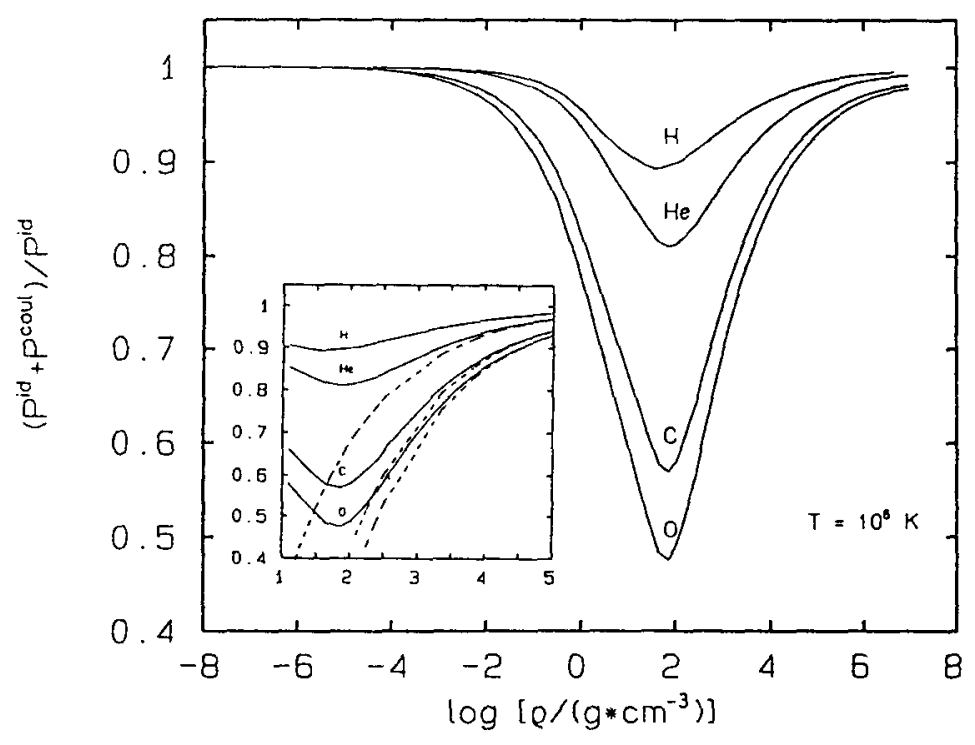

FIGURE I Relative pressure versus density for hydrogen, helium, carbon and oxygen at $T=10^{6} \mathrm{~K}$. The density range consider both the non-degenerate limit $(\log \rho<-2)$ and high-degenerate (relativistic) limit $(\log \rho>5)$. The inset shows the $T=0 \mathrm{~K}-$ EOS of Salpeter (1961) [dashed lines].

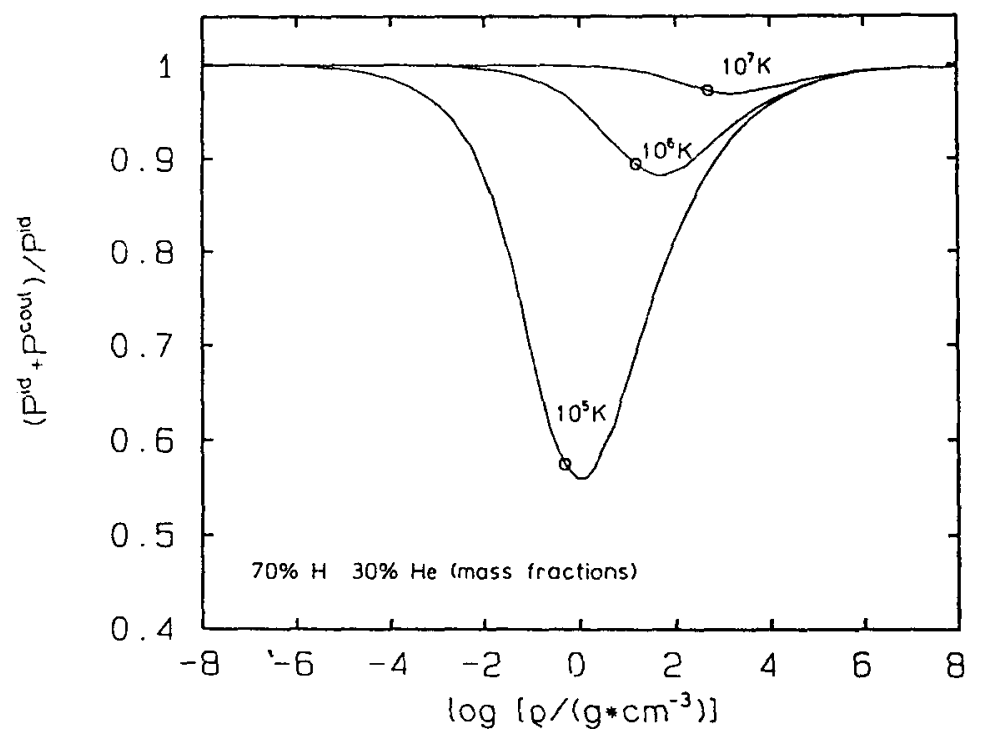

FIGURE II Relative pressure versus density for a $\mathrm{H} / \mathrm{He}$ mixture of $70 / 30$ by mass $\left(\approx 90 / 10\right.$ by particle numbers) for three isotherms of $T=10^{5}$, $10^{6}$ and $10^{7} \mathrm{~K}$. The circles illustrate typical $\rho, T$-values of a $0.2 M_{\odot}$ main sequence star according to D'Antona and Mazzitelli (1982). 
tion to the pressure, radiation pressure is not included.

The influence of the plasma composition is demonstrated in Fig. I. The strength of the Coulomb interaction increases strongly for heavy ions (e.g. carbon or oxygen). Three isotherms for a typical envelope composition of $\mathrm{H} / \mathrm{He}=$ $70 / 30$ by mass are presented in Fig. II. With decreasing temperature (at fixed density) the relative pressure decreases, too. Therefore, especially low-mass main sequence stars will be affected in their interiors by Coulomb interactions.

We have introduced a new formulation of the Coulomb interaction in the EOS which is valid in the whole temperature-density regime for fully ionized matter and applicable to arbitrary mixtures. Main features are:

- The major Coulomb contributions for high densities are the ion-ion interaction and the exchange term of the electrons.

- For low densities and moderate to high temperatures all considered contributions are of the same order of magnitude.

- Relativistic corrections influence the exchange term of the electrons significantly which becomes positiv for high densities $(\log \rho>6)$.

- The Coulomb interaction increases strongly for heavy elements.

- The new formulation provides a consistent connection between former low density/high temperature and high density/low temperature theories.

A detailed description of our investigations is given in Stolzmann and Blöcker (1992).

\section{REFERENCES}

Däppen, W., Hummer, D. G., Mihalas, D., Weibel-Mihalas, B.: 1988, ApJ 332, 261

D'Antona, F., Mazzitelli, I.: 1982, $A \mathscr{E} A$ 113, 303

Ebeling, W.: 1990, Contr. Plasma Phys. 30, 553

Ebeling, W., Richert, W., Kraeft, W. D., Stolzmann, W.: 1981, phys. stat. sol. (b) 104,193

Fontaine, G., Graboske, H. C., van Horn, H. M.: 1977, ApJS 35, 293

Graboske, H. C., Harwood Jr., D.J., Rogers, F. J.: 1969, Phys.Rev.A 186, 210

Hubbard, W. B., DeWitt, H. E.: 1985, ApJ 290, 388

Ichimaru, S., Iyetomi, H., Tanaka, S.: 1987, Phys. Rep. 149, 91

Kraeft, W. D., Kremp, D., Ebeling, W., Röpke, G.: 1986, Quantum statistics of charged particle systems, Plenum, New York

Magni, G., Mazzitelli, T.: 1979, $A \& A$ 72, 134

Salpeter, E. E.: 1961, ApJ 134, 669

Saumon, D., Chabrier, G.: 1991 Phys.Rev.A 44, 5122

Shaviv, G., Kovetz, A.: 1972, $A \& A$ 16, 72

Stolzmann, W., Blöcker, T.: 1992, $A \mathscr{E} A$, submitted 